Research Article

\title{
Detection of Biofilm production in microorganisms complicating Chronic wound infections
}

\author{
V. Abarna ${ }^{1}$, N. Sri Sakthipriya ${ }^{2}$, M. Anitha Raj ${ }^{3}$, E. Arthi ${ }^{4}$, Bagyalakshmi. R ${ }^{5}$, Jayalakshmi $G^{6}$ \\ ${ }^{1}$ Dr. V. Abarna, Assistant Professor, ${ }^{2}$ Dr. N. Sri Sakthipriya, Final year Medical Student, ${ }^{3}$ Dr. M. Anitha Raj, Assistant \\ Professor, ${ }^{4}$ Dr. E. Arthi, Assistant Professor, ${ }^{5}$ Dr. Bagyalakshmi. R, Assistant Professor, ${ }^{6}$ Dr. Jayalakshmi G. Professor, \\ all authors are affiliated with Department of Microbiology, Sri Lakshmi Narayana Institute of Medical Sciences, \\ Pondicherry, India.
}

Address for Correspondence: Dr. Abarna. V, Assistant Professor, Department of Microbiology, Sri Lakshmi Narayana Institute of Medical Sciences, Osudu, Agaram Village, Villianur Post, Pondicherry, South India. E- mail: dr.abarna@gmail.com

\begin{abstract}
Introduction: The presence of biofilm facilitates the development of infections by compromising the immune system of the patient and by contributing to the failure of antibiotic therapy, which may result in recurrent infections and the emergence of multidrug resistant pathogens. Objectives: The objective of this study is to determine the biofilm forming ability of the microorganisms infecting chronic wounds and to correlate the drug resistance with biofilm formation. Material and Methods: Wound swabs were collected from 50 patients with non-healing ulcers. Microorganisms isolated by standard microbiological techniques were screened for biofilm production by tissue culture plate method. Antibiotic sensitivity tests were done for appropriate panel of antibiotics by Kirby Bauer disc diffusion test. Results: All the 50 samples were culture positive. The most common organism isolated was Staphylococcus aureus, CoNS, Enterococcus faecalis, Beta hemolytic Streptococci, Pseudomonas aeruginosa, Escherichia coli, Klebsiella pneumonia, Acinetobacter and Proteus vulgaris. Biofilm formation was determined by Tissue Culture Plate method in 63 identified isolates (39 in monomicrobial \& 24 in polymicrobial infections) and $38(60.32 \%)$ isolates were positive for biofilm production. Most of the isolates in polymicrobial infections were strong biofilm producers. Biofilm producers were found to be more resistant to almost all the groups of antibiotics. Conclusion: If we understand the wound's microbial flora, it allows us to manage and treat the wound better. New strategies targeting biofilm disruption or prevention of biofilm formation are important new approaches in the management of chronic wounds.
\end{abstract}

Keywords: Biofilm, Chronic wound infection, Non healing ulcer.

\section{Introduction}

Chronic wound infections are accountable for substantial morbidity and drastically add to the increased cost of health care. Chronic wounds are defined as the one which does not heal in an expected way and taking longer duration for healing [1]. Wound infection primarily manifests as bacterial colonization over the ulcer. When this colonization is facilitated by other factors, like decreased vascular supply, compromised host immune status, specific intrinsic virulence factors in microorganisms, true infection

Manuscript received: $10^{\text {th }}$ September 2017

Reviewed: $20^{\text {th }}$ September 2017

Author Corrected: $28^{\text {th }}$ September 2017

Accepted for Publication: $3^{\text {rd }}$ October 2017 occurs [2,3]. Biofilm formation is one among the intrinsic virulence factor, exhibited by most of the bacteria, making the ulcers non-healable [4]. Microorganisms causing chronic wounds live within biofilm communities, in which the organisms are protected from host defense mechanisms and develop resistance to antibiotic treatment [4].

Formation of this biofilm and their inherent resistance to antimicrobial agents are the basis of many persistent and chronic wound infections. Therefore, recognition of biofilm producing organisms in non-healing ulcers continues to be a task that we should pursue diligently 


\section{Research Article}

and report as accurately and as rapidly as possible. The objective of this study is to determine the biofilm forming ability of the microorganisms infecting chronic wounds and to correlate the drug resistance with biofilm formation.

\section{Materials and Methods}

Study type \& place: This was a prospective study, which was carried out in two months duration from May 2015 to June 2015, in the Department of Microbiology, in Sri Lakshmi Narayana Institute of Medical Sciences, Pondicherry, South India. Wound swabs were collected from 50 patients with non-healing ulcers. Medical records for the source patients were searched for demographic information, history of prior hospitalization and presence of major conditions, (like Diabetes mellitus, renal dysfunctions, post surgical status, malignancy, trauma or burn injury).

Sample collection: Swabs were inoculated in routine culture media like Blood agar, MacConkey agar and incubated. The isolated organisms were identified by standard microbiological methods. Isolates were screened for biofilm production by tissue culture plate method. Antimicrobial susceptibility testing (appropriate panel of drugs for the identified isolates) were done on Muller Hinton agar by standard Kirby Bauer disc diffusion method and their susceptibilities to antimicrobials were recorded.

Detection of Biofilm production by Microtitre plate method [5, 6, 7]- Isolates were inoculated in brain heart infusion broth with $2 \%$ sucrose and were incubated overnight at $37^{\circ} \mathrm{C}$ and diluted further with 1 in 100 fresh BHI broth. $0.2 \mathrm{ml}$ of the diluted cultures was transferred to individual wells of sterile, polystyrene, 96 well-flat bottom microtitre plates. Uninoculated broth was used as control to check sterility and non-specific binding of media. Microtitre plates were incubated for 24 hours at $37^{\circ} \mathrm{C}$. Next day, the contents of each well were removed by gently tapping the plates. The wells were washed four times with $0.2 \mathrm{~mL}$ of saline to remove free-floating bacteria. Biofilms in plate were fixed with $2 \%$ sodium acetate and stained with $0.1 \%$ crystal violet. Optical density value (OD) of stained adherent bacteria was determined with ELISA reader at wavelength of $570 \mathrm{~nm}$. Experiments were performed in triplicate and repeated three times, the data were averaged and standard deviation was calculated. To compensate for background absorbance, OD readings from sterile medium, fixative and dye were averaged and subtracted from all the test values. The mean OD value obtained from media control well was deducted from all the test OD values (Table 1).

Table-1: Classification of bacterial adherence.

\begin{tabular}{|c|c|c|}
\hline Mean OD* value & Adherence & Biofilm formation \\
\hline$<0.120$ & Non & Non/weak \\
\hline $0.120-0.240$ & Moderately & Moderate \\
\hline$>0.240$ & Strong & High \\
\hline
\end{tabular}

*Optical density

\section{Results}

A total of 50 patients who was suffering from chronic wound infections were studied. Out of 50 patients, 14 (28\%) were females and $36(72 \%)$ were males. Sex and age wise distribution of the patients is given in Table 2 . Patients above 40 years were found to be affected by chronic wound infections.

While analyzing the diagnosis of patients, it was found that Diabetic foot -27 (54\%), Venous ulcers - 8 (16\%0, Post traumatic ulcers $-4(8 \%)$, Cellulitis $-4(6 \%)$, Non Union of Fracture $-3(6 \%)$, post operative infections $-3(6 \%)$, and infected implants $-1(2 \%)$ were the varying causes of non healing ulcer. (Table 3 )

When studying the risk factors like Diabetes, Hypertension, Smoking, Alcohol, History of previous hospitalization, Diabetes (in 31 patients) was found to be the most important precipitating factor among the other factors. 


\section{Research Article}

Table- 2: Age and Sex distribution of cases.

\begin{tabular}{|c|c|c|c|c|c|c|}
\hline \multirow{2}{*}{ Age group } & \multicolumn{2}{|c|}{ Males } & \multicolumn{2}{c|}{ Females } & \multicolumn{2}{c|}{ Total } \\
\cline { 2 - 7 } & No. & $\mathbf{\%}$ & No. & $\mathbf{\%}$ & No. & \% \\
\hline$<12 \mathrm{yrs}$ & 1 & 2 & 0 & 0 & 1 & 2 \\
\hline $12-40 \mathrm{yrs}$ & 12 & 24 & 6 & 12 & 18 & 36 \\
\hline$>40 \mathrm{yrs}$ & 23 & 46 & 8 & 16 & 31 & 62 \\
\hline Total & $\mathbf{3 6}$ & 72 & $\mathbf{1 4}$ & 28 & $\mathbf{5 0}$ & 100 \\
\hline
\end{tabular}

Table-3: Etiology of chronic ulcers.

\begin{tabular}{|c|c|c|}
\hline S.No & Causes of Chronic ulcers & No. of cases \\
\hline 1 & Diabetic foot & 27 \\
\hline 2 & Venous ulcer & 8 \\
\hline 3 & Post traumatic ulcer & 4 \\
\hline 4 & Cellulitis & 3 \\
\hline 5 & Non union of fracture & 3 \\
\hline 6 & Post operative infections & 1 \\
\hline 7 & Infected implants & 4 \\
\hline
\end{tabular}

Table-4: Distribution of Microbial flora in chronic wounds.

\begin{tabular}{|c|c|c|}
\hline S. No & Organisms isolated & Numbers \\
\hline 1 & Staphylococcus aureus & 17 \\
\hline 2 & CoNS & 10 \\
\hline 3 & Enterococcus faecalis & 7 \\
\hline 4 & Bata hemolytic Streptococci & 7 \\
\hline 5 & Pseudomonas aeruginosa & 6 \\
\hline 6 & Escherichia coli & 3 \\
\hline 7 & Klebsiella pneumoniae & 1 \\
\hline 8 & Acinetobacter spp. & 1 \\
\hline 9 & Proteus vulgaris & 8 \\
\hline
\end{tabular}

All the 50 samples were culture positive. 39 (78\%) samples were monomicrobial and 11 (22\%) samples were polymicrobial. The most common organism isolated was Staphylococcus aureus (17), followed by, Coagulase Negative Staphylococcus (10), Enterococcus faecalis (8), Beta hemolytic Streptococci (7), Gram negative bacteria like, Pseudomonas aeruginosa (7), Escherichia coli (6), Klebsiella pneumoniae (3), Acinetobacter (1) and Proteus vulgaris (1). (Table 4).

Almost all polymicrobial infection revealed presence of Staphylococcus aureus, either in combination with a gram negative bacilli or a beta hemolytic Streptococci.

Biofilm formation was determined by Tissue Culture Plate method in 63 identified isolates (39 in monomicrobial \& 24 in polymicrobial infections) and $38(60.32 \%)$ isolates were positive for biofilm production.

In TCP method, among 63 isolates tested for biofilm formation, strong biofilm producers were $23(36.52 \%), 15(23.81 \%)$ were moderate and 25 (39.68\%) isolates were considered as non or weak biofilm producers. 


\section{Research Article}

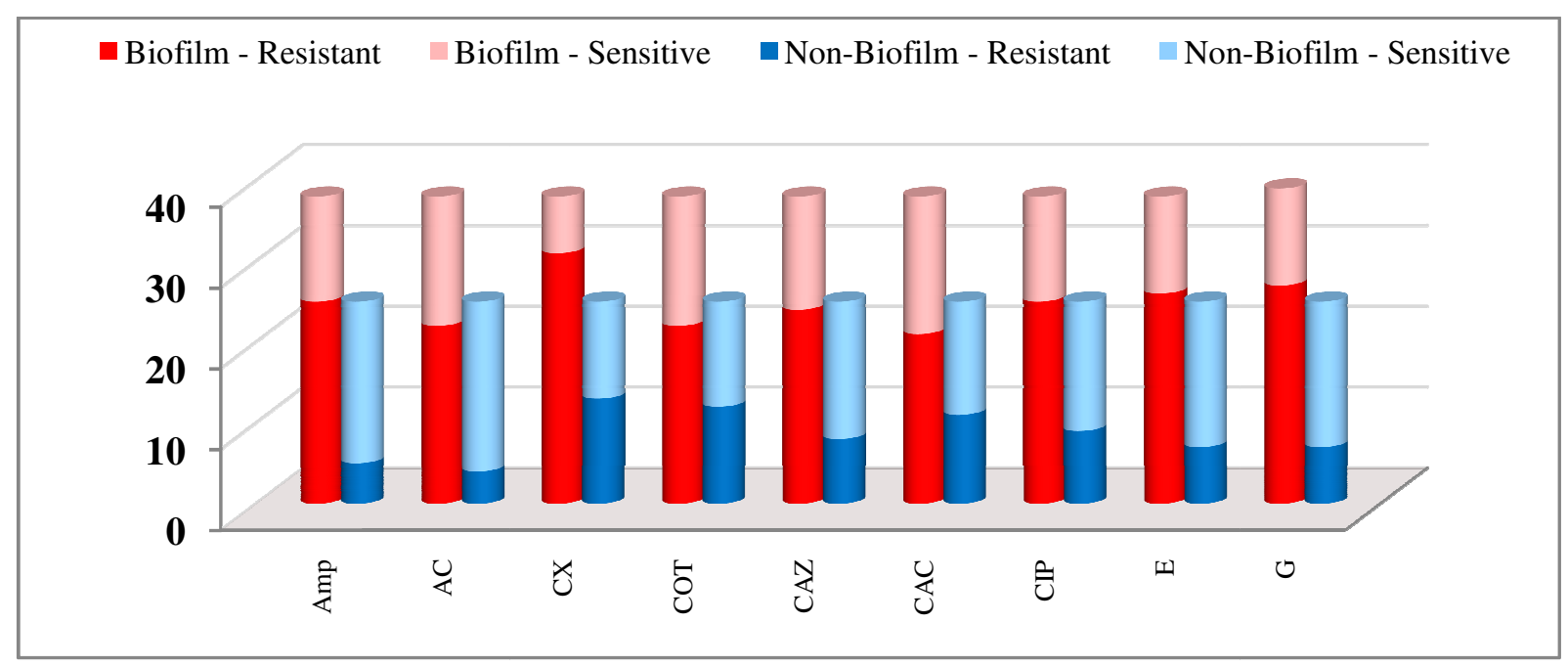

Figure-1: Antibiotic Sensitivity Pattern in Bio-film Producing \& Non-Bio film producing Organisms

Biofilm producers were found to be more resistant to almost all the groups of antibiotics (Figure 1). Most of the isolates in polymicrobial infections were strong biofilm producers.

\section{Discussion}

The presence of biofilm facilitates the development of infections by compromising the immune system of the patient and by contributing to the failure of antibiotic therapy, which may result in recurrent infections and the emergence of multidrug resistant pathogens. The ability to form biofilm on wounds is a potential risk factor for its chronicity. There was a significant difference between different age groups infected with chronic wounds. Elderly patients ( $>40$ years of age) were significantly more prone for infections as compared to children and young adults in our study. This might be because elderly are more immunocompromised and are affected by debilitating illnesses like diabetes, more than the young people.

Diabetics are at a higher risk of chronic infections. In our study, chronic wound infections were very high among diabetics $(87.01 \%)$, which is accordance with other studies. Diabetic foot infections should be treated aggressively, since the host is immune-suppressed. Maintaining healthy blood levels, Monitoring cuts, abrasions, rashes, and other breaks in the skin, for infection, will prevent chronic infections in diabetics.

Various studies identify Staphylococcus spp. as the predominant organisms associated with acute and chronic wounds [1-3, 8]. Similarly, in our study also Staphylococcus aureus was the predominant organism isolated from the chronic wounds. Other organisms that have been most commonly identified in association with chronic wounds include Pseudomonas sp, E. coli, Klebsiella pneumoniae, Streptococcus, Enterococcus spp and Proteus spp. Sixty percent of chronic wound specimens were characterized as containing biofilm, whereas only $6 \%$ of acute wounds contained biofilm, indicating biofilms were prevalent in chronic wound samples and relatively rare in samples from acute wounds [9]. This is in accordance with our study in which $60.32 \%$ of identified isolates were biofilm producers. And few studies have shown relatively low prevalence also. This variation might be because of several factors like efficacy of infection control practices, healthcare facilities and antibiotic usage that vary from hospital to hospital.

Notably, in nosocomial infections, biofilm provides an excellent and relatively protected growth medium for microorganisms. Almost all the Staphylococcal species (17 Staphylococcus aureus and 10 CoNS) were biofilm producer. Staphylococcal species themselves contributes to and promotes the formation of biofilm, which, in turn, facilitates the transfer of genetic material conferring resistance between species.

Mathur et al stated that Tissue culture plate method is an accurate and reproducible method for screening $\&$ it is a reliable quantitative tool for determining biofilm formation by clinical isolates [5]. 


\section{Research Article}

Biofilm producers were found to be more resistant to almost all the groups of antibiotics. Biofilm consists of multilayered cell clusters embedded in a matrix of extracellular polysaccharide (slime), which facilitates the adherence of these microorganisms to surfaces and protect them from host immune system and antimicrobial therapy. It is now well documented that biofilms are notoriously difficult to eradicate and are often resistant to systemic antibiotic therapy [10-12].

Hence along with antibiotics, biofilm based wound care is essential in Staphylococcal infections. Multiple Concurrent Strategies in Biofilm based wound care is essential, which include, Frequent and aggressive Debridement, use of selective biocides (Silver, Iodosorb, Hydrofera Blue) and use of Antibiofilm Agents like Lactoferrin, Xylitol, Farnasol, Plant Products, Fatty Acid Gel.

Biofilm formation is regulated by expression of polysaccharide intracellular adhesin (PIA), which mediates cell to cell adhesion and is the gene product of icaADBC. Further expansion of this study can be done by analyzing the gene (icaADBC) for molecular confirmation of biofilm production. Limitation of this study is the smaller sample size with which appropriate statistical analysis cannot be made.

\section{Conclusion}

Bacteria living in a biofilm are relatively resistant to antibiotics than free living bacteria and the biofilms are usually polymicrobial. This explains why antibiotics alone are of limited use in treating chronic wounds. New strategies targeting biofilm disruption or prevention of biofilm formation are important new approaches in the management of chronic wounds. If we understand the wound's microbial flora, it allows us to manage and treat the wound better.

The bacterial population within a pathogenic biofilm provides the bacterial community as a whole with an enhanced ability to persist and thrive in a variety of antagonistic situations, even in spite of combined host and antibiotic attack.

Identification of the factors affecting the evolution from colonization to infection can help surgeons in treating the patients with chronic wounds. Appreciation of the physiology and protective mechanisms of biofilms may lead to the development of more effective methods in treating non healing ulcers.

Acknowledgement- The authors are thankful to Indian Council of Medical Research (ICMR) for approving this project under Short Term Studentship (ICMR STS) in 2015.

Funding: Nil, Conflict of interest: None initiated, Permission from IRB: Yes

\section{References}

1. Urbancic-Rovan, V. and Gubina, M. (2000), Bacteria in superficial diabetic foot ulcers. Diabetic Medicine, 17: 814-815. doi:10.1046/j.1464-5491.2000.00374-2.x.

2. Bowler PG, Davies BJ. The microbiology of infected and noninfected leg ulcers. Int J Dermatol. 1999 Aug; 38 (8):573-8.

3. James GA, Swogger E, Wolcott R, Pulcini Ed, Secor P, Sestrich J, Costerton JW, Stewart PS. Biofilms in chronic wounds. Wound Repair Regen. 2008 Jan-Feb; 16(1): 37-44. Epub 2007 Dec 13.

4. Brown MR, Allison DG, Gilbert P. Resistance of bacterial biofilms to antibiotics: a growth-rate related effect? J Antimicrob Chemother. 1988 Dec; 22(6): 777-80.

5. Mathur T, Singhal S, Khan S, Upadhyay DJ, Fatma $\mathrm{T}$, Rattan A. Detection of biofilm formation among the clinical isolates of Staphylococci: an evaluation of three different screening methods. Indian J Med Microbiol. 2006 Jan;24(1):25-9.

6. Christensen GD, Simpson WA, Younger JA et al. (1995) Adherence of coagulase negative Staphylococci to plastic tissue cultures: a quantitative model for the adherence of Staphylococci to medical devices. J Clin Microbiol. 1985 Dec; 22(6): 996-1006.

7. Hassan A, Usman J, Kaleem F, Omair M, Khalid A, Iqbal M. Evaluation of different detection methods of biofilm formation in the clinical isolates. Braz $\mathrm{J}$ Infect Dis. 2011 Jul-Aug;15(4):305-11.

8. Brook I, Frazier EH. Aerobic and anaerobic microbiology of chronic venous ulcers. Int J Dermatol. 1998 Jun;37(6):426-8. 


\section{Research Article}

9. Costerton W, Veeh R, Shirtliff M, Pasmore M, Post C, Ehrlich G. The application of biofilm science to the study and control of chronic bacterial infections. J Clin Invest. 2003 Nov;112(10):1466-77.

10. Anwar, H., Strap, J.L., and Costerton, J.W. (1992) Establishment of aging biofilms: a possible mechanism of bacterial resistance to antimicrobial therapy. AntimicrobAgentsChemother.1992Jul;36(7):13471351.
11. Brown MR, Allison DG, Gilbert P. Resistance of bacterial biofilms to antibiotics: a growth-rate related effect? J Antimicrob Chemother. 1988 Dec; 22 (6): 777-80.

12. Stewart PS, Costerton JW. Antibiotic resistance of bacteria in biofilms. Lancet. 2001 Jul 14; 358 (9276) : 135-8.

\section{How to cite this article?}

V. Abarna, N. Sri Sakthipriya, M. Anitha Raj, E. Arthi, Bagyalakshmi. R, Jayalakshmi G. Detection of Biofilm production in microorganisms complicating Chronic wound infections. Pathology Updare: Trop J Path Micro 2017;3(4):362-367.doi:10.17511/jopm.2017.i4.01. 